\title{
The Glycemic Control Difference in Type 1 and Type 2 Diabetic Patients
}

\author{
Yasemin Sefika Akdeniz ${ }^{1 *}$, Hamide Piskinpasa1 ${ }^{1}$, Ayse Esen ${ }^{1}$, Ozlem Polat ${ }^{2}$, Isil Ozbas Tevetoglu ${ }^{3}$, Gokturk Ogredici ${ }^{3}$, \\ Yildiz Okuturlar ${ }^{4}$, Sema Ciftci Dogansen ${ }^{1}$, Pinar Karakaya ${ }^{1}$ and Meral Mert ${ }^{1}$
}

${ }^{1}$ Department of Endocrinology and Metabolism, University of Health Sciences, Turkey

${ }^{2}$ Department of Family Medicine, University of Health Sciences, Turkey

${ }^{3}$ Department of Internal Medicine, University of Health Sciences, Turkey

${ }^{4}$ Department of Internal Medicine, University of Acibadem, Turkey

*Corresponding author: Yasemin Sefika Akdeniz, Department of Endocrinology and Metabolism, University of Health Sciences, Bakirkoy Dr. Sadi Konuk Training and Research Hospital, Turkey

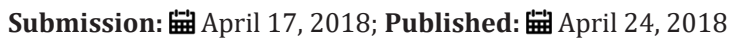

\begin{abstract}
Effective treatment and follow-up for type 1 diabetics have resulted significant increase in the number of adult type 1 diabetic patients. Psychological adaptation problem and impairment in glycemic control have been observed Type 1 diabetic patients that have been referred from pediatric endocrinology clinics to adult endocrinology clinics. Type 1 and Type 2 diabetes are clinically different from each other in terms of age of onset, ketosis tendencies, family history and metabolic differences. We investigated whether there was a difference between glycemic controls of type 1 and type 2 diabetic patients who were referred to our hospital endocrinology and diabetes outpatient clinics, and probable causes for his condition. 17,985 patients who applied to our hospital for the last 5 years were included. Age, sex, glucose, A1C, triglyceride, total cholesterol, LDL, HDL and TSH levels of all patients were recorded retrospectively. Patients were divided into two groups, Type 1 and Type 2 diabetes.
\end{abstract}

Glucose ( $\mathrm{p}=0.0001)$ and $\mathrm{A} 1 \mathrm{C}(\mathrm{p}=0.0001)$ values were found to be statistically higher in type 1 diabetic patients. In Type 2 diabetic patients total cholesterol, LDL cholesterol and triglycerides (TG) levels were significantly higher, while there was no statistically significant difference in HDL and TSH levels. Oral anti-diabetic use rather than frequent insulin injections in our Type 2 diabetes group may be one of the factors that increase treatment compliance. Relative high incidence of hypoglycemia due to absolute insulin deficiency in type 1 diabetics may lead to weight problems and increase in $\mathrm{A} 1 \mathrm{C}$ as the dose of insulin may be skipped. In type 1 diabetics, prolonged duration of the disease may be one of the factors that leads to impaired glycemic control and increased diabetic complications

Keywords: Type 1 diabetes mellitus; Type 2 diabetes mellitus; Glycemic control; A1c

\section{Introduction}

Type 1 and type 2 diabetes are heterogeneous diseases in terms of clinical onset patterns and prognosis. It has been assumed that type 1 diabetes begins with acute hyperglycemia or diabetic ketoacidosis (DKA) in children and adolescents, whereas type 2 diabetes begins with mild and relatively slow course in adults. There is a reduction of $\beta$-cell mass and function both in type 1 and type 2 diabetes due to genetic and environmental factors [1,2]. In type 1 diabetes which usually starts before the age of 30 , the cause is an absolute deficiency of insulin secretion. Type 1 diabetes is ketone prone diabetes. Symptoms such as polydipsia, polyphagia, polyuria, weight loss and fatigue suddenly appear in type 1 diabetic. Type 1 diabetics are often underweight or normal weight [3].

Decreased insulin sensitivity and decreased glucose uptake due to post-receptor cell deficiency leading intracellular hypoglycemia play very important role in type 2 DM pathophysiology. Type- 2 diabetes present with diminished glucose transport and disposal especially in muscles and adipose tissue. Insulin resistance begins before Type- 2 diabetes and it dominates the picture for many years. On the other hand, an abrupt decrease in insulin secretion may occur during the later stages of diabetes or in case of intervening diseases. Type- 2 diabetes usually occurs after the age of 30. Especially over the last 10-15 years as a consequence of the increased incidence of obesity, type-2 DM is more common in childhood and adolescence. There is a strong genetic predisposition. The stronger family history gets, the greater risk of diabetes in subsequent generations and the disease begins to appear earlier in life. Patients are often obese or have a high BMI [Body mass index (BMI)> 25kg/m2]. Actually patients are not prone to diabetic ketoacidocis. However, DKA may be seen in prolonged periods of hyperglycemia or when beta cell reserve declines. The onset of Type 2 diabetes is insidious and is usually recognized only 5-12 years after hyperglycemia develops. Many patients have no symptoms at initial period of Type 2 DM. Some patients may present in outpatient clinic with blurred vision, 
numbness and tingling in the hands and feet, foot pain, recurrent fungal infections or delayed wound healing before diagnosis of type-2 DM [1,3].

Effective treatment and follow-up for type 1 diabetics have resulted significant increase in the number of adult type-1 diabetic patients. Psychological adaptation problem and impairment in glycemic control have been observed Type- 1 diabetic patients that have been referred from pediatric endocrinology clinics to adult endocrinology clinics [4]. Type 1 and Type 2 diabetes are clinically different from each other in terms of age of onset, ketosis tendencies, family history, and metabolic differences. We investigated whether there was a difference between glycemic controls of type 1 and type 2 diabetic patients who were referred to our hospital endocrinology and diabetes outpatient clinics, and probable causes for his condition.

\section{Materials and Methods}

17,985 patients who applied to our hospital for the last 5 years were included. Age, sex, glucose, A1C, triglyceride, total cholesterol, LDL, HDL and TSH levels of all patients were recorded retrospectively. Patients were divided into two groups, Type 1 and
Type 2 diabetes. Blood samples are taken following 12 hour fasting, put into gelled dry tubes and left for approximately 30 minutes and then centrifuged at $4000 \mathrm{rpm}$ for 10 minutes. Biochemical analyzes were performed on the same day using original kits on Beckman Coulter brand AU5800 model biochemical auto-analyzer (Beckman Coulter inc., U.S.A). HbA1c were put into EDTA tubes, using HPLC (High performance liquid chromatography) and Arkray Adam HA$8180 \mathrm{~V}$ model analyzer. SPSS 17 was used as a statistical method.

\section{Results and Discussion}

In Type 1 (2.8\%) diabetic patients, 270 (54.1\%) were female, 229 (45.9\%) were male; while in Type 2 (97.2\%) diabetic patients, 11126 (63.6\%) were female, 6360 (36.4\%) were male Figure 1. Results can be seen in Table 1 . Glucose $(p=0.0001)$ and A1C $(\mathrm{p}=0.0001)$ values were found to be statistically higher in type 1 diabetic patients. In Type 2 diabetic patients total cholesterol, LDL cholesterol and TG levels were significantly higher, while there was no statistically significant difference in HDL and TSH levels. 54.1\% of Type $1 \mathrm{DM}$ patients and $63.6 \%$ of Type 2 DM patients were female. Statistically significant difference was found between these two groups $(\mathrm{p}=0.0001)$.

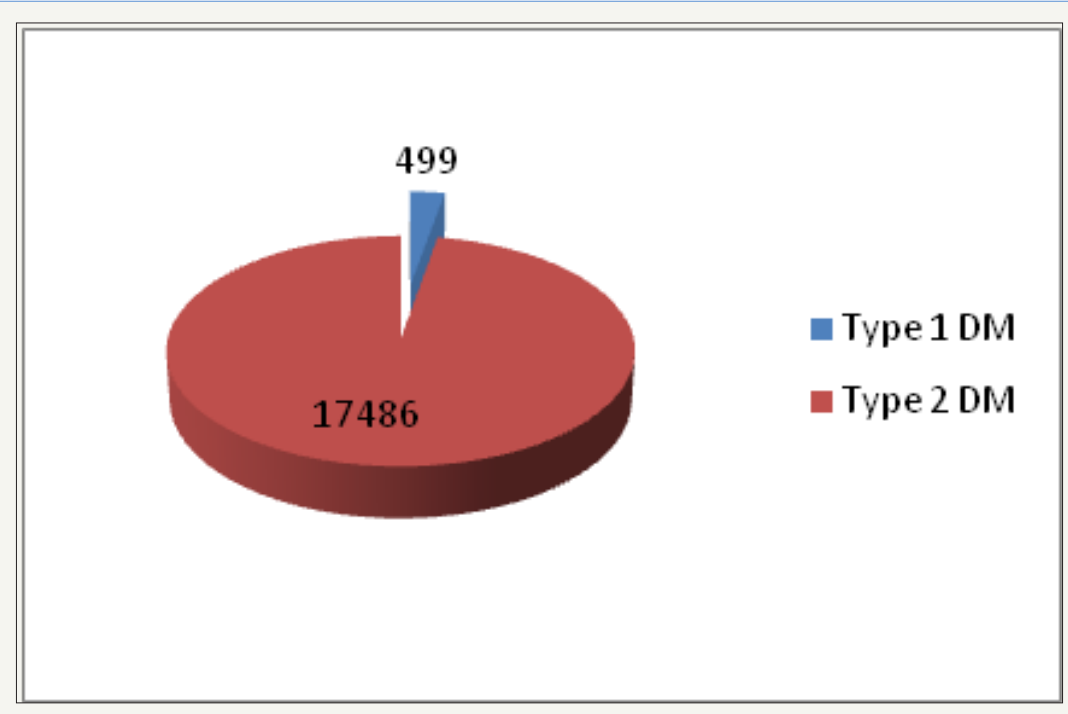

Figure 1: Type 1 and 2 diabetic patients.

Table 1: The difference between glycemic control of type 1 and type 2 diabetic patients.

\begin{tabular}{|c|c|c|c|c|c|c|c|}
\hline \multirow{2}{*}{$\mathbf{n}=\mathbf{1 7 9 8 5}$} & \multicolumn{3}{|c|}{ Type 1 n=499 } & \multicolumn{3}{c|}{ Type 2 n=17486 } & \multirow{2}{*}{ p Value } \\
\cline { 2 - 8 } & Mean \pm SD & Median & Min-Max & Mean \pm SD & Median & Min-Max & $18-106$ \\
\hline Age (year) & $46.64 \pm 17.51$ & 49.00 & $18-89$ & $55.29 \pm 12.82$ & 56.00 & 0.0001 \\
\hline Glycose (mg/dl) & $201.70 \pm 97.1$ & 174.00 & $42-545$ & $155.92 \pm 75.10$ & 129.00 & $22-708$ & 0.0001 \\
\hline Hb A1c & $8.80 \pm 2.26$ & 8.50 & $5.1-17.8$ & $7.47 \pm 2.05$ & 6.80 & $5-17.09$ & 0.0001 \\
\hline Total cholesterol & $195.23 \pm 45.83$ & 191.00 & $98-419$ & $208.03 \pm 48.28$ & 205.00 & $66-1089$ & 0.0001 \\
\hline Trigliserid & $157.24 \pm 125.85$ & 128.00 & $38-1347$ & $175.29 \pm 148.21$ & 144.00 & $18-9283$ & 0.0001 \\
\hline HDL cholesterol & $50.38 \pm 15.88$ & 48.00 & $6-116$ & $48.78 \pm 14.35$ & 47.00 & $2-152$ & 0.103 \\
\hline LDL cholesterol & $119.01 \pm 39.09$ & 115.20 & $23.2-345$ & $128.13 \pm 40.36$ & 125.85 & $6-466$ & 0.0001 \\
\hline TSH & $2.83 \pm 5.46$ & 2.05 & $0.02-61$ & $2.75 \pm 5.68$ & 1.88 & $0.005-165$ & 0.626 \\
\hline
\end{tabular}

SD: Standard Deviation; Min: Minimum; Max: Maximum; Hb A1C: Glycosylated Hemoglobin; HDL: High Density Lipoprotein; LDL: Low Density Lipoprotein; TSH: Thyroid Stimulating Hormone 


\section{Discussion}

A1C reflects average glycemia over approximately 3 months and has strong predictive value for diabetes complications. Thus, A1C testing should be performed routinely in all patients with diabetes at initial assessment and as part of continuing care. Measurement approximately every 3 months determines whether patients' glycemic targets have been reached and maintained. It's a test to assess glycemic control [5]. T1DM is an autoimmune disease caused by the interaction of genetic, environmental and immunological factors. The traditional concept is that some environmental factors can trigger an immune response against pancreatic B cells in genetically susceptible individuals [6]. It is well known that glycemic control is influenced by the emotional, psychological, behavioral and socioeconomic factors that influence treatment compliance in the long-term course of T1DM [7]. Also studies have suggested that before the onset of chronic complications, patients with T1DM experience relatively small reductions in their healthrelated quality of life (HRQOL). Studies examining the effects of treatment, including comparisons of insulin type, frequency of injections, and pump use, have not shown consistent effects on HRQOL [8-12].

Similarly, variations in the level of glycemic control and/or exposure to severe hypoglycemia were not consistently associated with HRQOL level [13]. A small number of largely crosssectional studies have indicated that diabetes complications are more strongly and consistently associated with lower quality of life [14]. Consistent with prevalence studies, in our study, type 1 diabetic patients is outnumbered by type 2 diabetic patients. Although Type 1 diabetics are younger, they appear to be worse in glycemic control. Due to the fact that Type $1 \mathrm{DMs}$ are diagnosed at a younger age, the duration of diabetes may be longer, compliance problems may develop. Psychosomatic incompatibilities following diabetes, starting at an earlier age, can be an important factor in terms of micro and macro-vascular complications that may occur in the process. Frequent follow-up, long-term medical and psychosocial support may be more important in diabetic patients during childhood and adolescence.

T1DM and T2DM have different underlying pathophysiology. T1DM is usually characterized by early onset of insulin-producing cells leading to insulin deficiency, which is the underlying cause of autoimmunity and destruction. In contrast, T2DM is characterized by the onset of an adult hyperinsulinemia as a consequence of insulin resistance and the slow progression of hyperglycaemia. T2DM is associated with obesity and its incidence increases with age $[15,16]$. Holzman et al. [17] showed that, T1DM was associated with a doubling of mortality, but also that patients with T2DM had only a minimally increased risk of death in comparison with nondiabetic patients after coronary by pass-grefting surgery (CABG) [17]. Also they found that, patients with T1DM were more likely than patients with T2DM or without diabetes to have comorbidities, such as chronic kidney disease, end-stage renal disease, peripheral vascular disease, or heart failure, which all have been associated with a worse prognosis in diabetic patients who undergo CABG [17].

Similarly Olafson et al. [18] found that HbA1c levels were higher in patients with T1DM than in those with T2DM, revealing poorer glycemic control in patients with T1DM. The association between glycemic control and micro- and macroangiopathy seems to be more significant in T1DM than in T2DM $[18,19]$. Oral antidiabetic use rather than frequent insulin injections in our Type 2 diabetes group may be one of the factors that increase treatment compliance. Relative high incidence of hypoglycemia due to absolute insulin deficiency in type 1 diabetics may lead to weight problems and increase in $\mathrm{A} 1 \mathrm{C}$ as the dose of insulin may be skipped. In type 1 diabetics, prolonged duration of the disease may be one of the factors that leads to impaired glycemic control and increased diabetic complications. This study was retrospective, and type 2 diabetics using oral anti-diabetics weren't excluded. These are our study's limitations. We believe that future studies that compares Type 1 and Type 2 diabetic patients just using insulin therapy in the same age group, will be shown more details.

\section{Conclusion}

In type 1 diabetics, prolonged duration of the disease may be one of the factors that leads to impaired glycemic control and increased diabetic complications. Moreover, in type 2 diabetics, oral anti-diabetic use rather than frequent insulin injections may be one of the factors that increase treatment compliance.

\section{References}

1. American Diabetes Association (2018) Classification and diagnosis of diabetes: standards of medical care in diabetes-2018. Diabetes Care 41(Suppl 1): S13-S27.

2. Dabelea D, Rewers A, Stafford JM, Standiford DA, Lawrence JM, et al. (2014) Trends in the prevalence of ketoacidosis at diabetes diagnosis: the SEARCH for diabetes in youth study. Pediatrics 133(4): e938-e945.

3. Satman I (2010) Clinical practice guidelines for diagnosis,treatment and follow-up of Diabetes Mellitus and its complications. Turkish Journal of Endocrinology and Metabolism 14(12): 1-7.

4. Lotstein DS, Seid M, Klingensmith G, Case D, Lawrence JM, et al. (2013) Transition from pediatric to adult care for youth diagnosed with type 1 diabetes in adolescence. Pediatrics 131(4): e1062-e1070.

5. International Expert Committee (2009) International expert committee report on the role of the a1c assay in the diagnosis of diabetes international expert committee. Diabetes Care 32(7): 1327-1334.

6. De Block CE, De Leeuw IH, Vertommen JJ, Rooman RP, Du Caju MV, et al. (2001) Beta-cell, thyroid, gastric, adrenal and coeliac autoimmunity and HLA-DQ types in type 1 diabetes. Clin Exp Immunol 126(2): 236-241.

7. Shalitin S, Phillip M (2012) Which factors predict glycemic control in children diagnosed with type 1 diabetes before 6.5 years of age? Acta Diabetol 49(5): 355-362.

8. Jacobson AM (2004) Impact of improved glycemic control on quality of life in patients with diabetes. Endocr Pract 10(6): 502-508.

9. Diabetes Control and Complications Trial Research Group (1996) Influence of intensive diabetes treatment on quality-of-life outcomes in the diabetes control and complications trial. Diabetes Care 19(3): 195203. 
10. Lawrence JM, Yi-Frazier JP, Black MH, Anderson A, Hood K, et al. (2012) Demographic and clinical correlates of diabetes-related quality of life among youth with type 1 diabetes. J Pediatr 161(2): 201-207.

11. Huang ES, Brown SES, Ewigman BG, Foley EC, Meltzer DO (2007) Patient perceptions of quality of life with diabetes-related complications and treatments. Diabetes Care 30(10): 2478-2483.

12. Khanna A, Bush AL, Swint JM, Peskin MF, Street RL, et al. (2012) Hemoglobin A1c improvements and better diabetes-specific quality of life among participants completing diabetes self-management programs: a nested cohort study. Health Qual Life Outcomes 10: 48.

13. Rubin RR, Peyrot M, STAR 3 Study Group (2012) Health-related quality of life and treatment satisfaction in the SensorAugmented Pump Therapy for A1C Reduction 3 (STAR 3) trial. Diabetes Technol Ther 14(2): 143151.

14. Fenwick EK, Xie J, Ratcliffe J, Pesudovs K, Finger RP, et al. (2012) The impact of diabetic retinopathy and diabetic macular edema on healthrelated quality of life in type 1 and type 2 diabetes. Invest Ophthalmol Vis Sci 53(2): 677-684
15. Laakso M, Kuusisto J (2014) Insulin resistance and hyperglycaemia in cardiovascular disease development. Nat Rev Endocrinol 10(5): 293302.

16. Defronzo RA (2009) Banting Lecture. From the triumvirate to the ominous octet: a new paradigm for the treatment of type 2 diabetes mellitus. Diabetes 58(4): 773-795.

17. Holzmann MJ, Rathsman B, Eliasson B, Kuhl J, Svensson AM, et al. (2015) Long-term prognosis in patients with type 1 and 2 diabetes mellitus after coronary artery bypass grafting. J Am Coll Cardiol 65(16): 16441652.

18. Eeg-Olofsson K, Cederholm J, Nilsson PM, Zethelius B, Svensson AM, et al. (2010) Glycemic control and cardiovascular disease in 7,454 patients with type 1 diabetes: an observational study from the Swedish National Diabetes Register (NDR). Diabetes Care 33(7): 1640-1646.

19. Juutilainen A, Lehto S, Ronnemaa T, Pyorala K, Laakso M (2008) Similarity of the impact of type 1 and type 2 diabetes on cardiovascular mortality in middle-aged subjects. Diabetes Care 31(4): 714-719.
Creative Commons Attribution 4.0 International License

For possible submissions Click Here

\section{Submit Article}

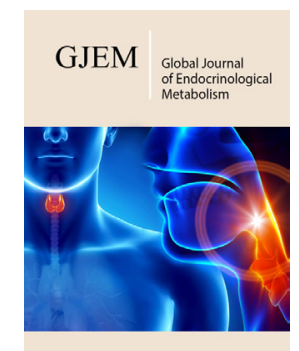

Global Journal of Endocrinological Metabolism

\section{Benefits of Publishing with us}

- High-level peer review and editorial services

- Freely accessible online immediately upon publication

- Authors retain the copyright to their work

- Licensing it under a Creative Commons license

- Visibility through different online platforms 